\title{
Ultrasound assisted catheter directed thrombolysis and aspiration thrombectomy for acute limb ischemia caused by embolization of infective endocarditis vegetations
}

\author{
Kevin Kang ${ }^{1 *}$, Robert Maholic ${ }^{2}$ and Gurjaipal S Kang ${ }^{2}$ \\ ${ }^{1}$ Dartmouth Geisel School of Medicine, Hanover, NH 03755, USA \\ ${ }^{2}$ UPMC Hamot Hospital, Erie, PA, USA
}

\begin{abstract}
Background: Endocarditis can cause peripheral emboli leading to acute limb ischemia (ALI). The standard of care for ALI from Infective Endocarditis (IE) is not established.

Case Presentation: 19-year-old male with mitral valve IE had acute bilateral limb ischemia. On the left leg, the angiograms showed occlusion of iliac and superficial femoral (SFA) arteries. These lesions were unsuccessfully treated with balloon angioplasty but overnight Ultrasound catheter directed thrombolysis or Ekos thrombolysis (Ekos corporation) given at $24 \mathrm{mg}$ of tissue plasminogen activator for 12 hours resulted in patency of SFA and Iliac arteries. However, the profunda femoris artery (PFA) became occluded due to embolization after Ekos lysis. Balloon angioplasty of PFA was unsuccessful and Rheolytic thrombectomy failed as well but direct aspiration of the vegetation material with a 7F guiding catheter was successful and the PFA patency was recovered, however there was distal embolization resulted in occlusion of the distal popliteal artery. Aspiration thrombectomy was successful in causing one vessel patency to the foot. The right leg had occluded PFA and popliteal artery and balloon angioplasty failed but again an excellent response to overnight Ekos thrombolysis was seen with patency of popliteal artery. The aspiration material was sent to pathology laboratory and showed IE emboli.
\end{abstract}

The patient had a good angiographic and clinical result from percutaneous ultrasound catheter directed thrombolysis. The mitral valve needed to be replaced but the lower extremities had no further clinical sequelae over 6 months of follow up.

Conclusions: IE septic emboli may be treated with ultrasound enhanced catheter directed thrombolysis and also by aspiration thrombectomy. Balloon angioplasty alone led to poor outcomes in our patient.

\begin{abstract}
Abbreviations: ALI: Acute limb ischemia; IE: Infective endocarditis; SFA: Superficial femoral artery; PFA: Profunda femoris artery; tPA: Tissue plasminogen activator
\end{abstract}

\section{Background}

We did not find a similar study published in the literature where septic emboli to lower extremities were treated with ultrasound facilitated thrombolysis followed by aspiration thrombectomy with success

\section{Case presentation}

Infective Endocarditis (IE) has an incidence of about 10 episodes per 100,000 person-years. 1 IE, most commonly, occurs on valvular or nonvalvular cardiac structures with underlying structural abnormality that results in blood flow turbulence, endothelial disruption and platelet and fibrin deposition. The resulting lesion is called nonbacterial thrombotic endocarditis suggesting the strong thrombotic component of the lesion and serves as a nidus for adhesion by bacteria or fungi. This is the predominant mechanism of IE and usually affects left sided valves. IE affects the left-sided valves more often than the right-sided valves, affecting the aortic valve in $38 \%$ and the mitral valve in $41 \%$ of cases. Embolic events are common early in IE course especially before antibiotic therapy is initiated. The bulky valvular and nonvavular vegetations can break off: systemic septic emboli occur in $20-50 \%$ of native valve IE patients and are more common in younger patients. The presence of large mobile vegetations on the echocardiogram particularly on the anterior mitral leaflet predicts high risk of embolic complications [1]. Acute limb ischemia (ALI) is a common vascular emergency and is usually due to underlying peripheral vascular disease and thrombotic emboli but lower limb ALI can occur as a consequence of septic embolic events in IE [1,2]. Although the incidence of limb ischemia in IE is not extensively studied, two case series reveal that about $5-8 \%$ of IE patients get peripheral ischemic syndromes including ALI $[1,3]$.

A 19-year-old male with history of Facioscapulohumeral dystrophy presented with a 2-month history of fever, chills, chest pains and had positive troponin-I $(5 \mathrm{ng} / \mathrm{ml})$ and sinus tachycardia. His blood cultures revealed Streptococcus viridans and his echocardiogram showed a 3 $\mathrm{cm}$ vegetation on a myxomatous mitral valve. By the next afternoon,

${ }^{\star}$ Correspondence to: Kang K, BA, MD candidate, Dartmouth Geisel School Of Medicine, Hanover, NH 03755, Tel: 814-450-8160; Fax: 814-450-8160; E-mail: kevinsinghkang@gmail.com

Key words: peripheral vascular disease, peripheral interventions, thrombolytic therapy, critical limb ischemia

Received: May 28, 2020; Accepted: June 13, 2020; Published: June 16, 2020 
Kang K (2020) Ultrasound assisted catheter directed thrombolysis and aspiration thrombectomy for acute limb ischemia caused by embolization of infective endocarditis vegetations

he developed new onset bilateral lower extremity pain with left leg, colder and more painful than his right leg. He had lost pulses over both feet and also over the left femoral area and was diagnosed with IE related emboli, especially severe on the left side. He was taken to the Cardiac Catheterization Laboratory where bilateral lower extremity angiography Digital Subtraction angiogram was performed from right common femoral access by placing 5 French (F) Verrill Contralateral Flush or VCF catheter (Cook Medical) at the abdominal aorta.

This revealed occlusive lesions in both lower extremities. On the left side, total occlusion of the left common iliac at its site of bifurcation to internal and external iliac arteries was noted with no distal filling beyond the occlusion (Figure 1). On the right side, there was occlusion of the proximal profunda femoris artery (PFA) and at the distal popliteal, there was a hazy filling defect with brisk filling of anterior tibial artery via collaterals but poor filling of peroneal and posterior tibial.

The left leg was, clinically, much worse than the right and the percutaneous intervention to the right leg was deferred and the left leg was targeted first. The tip of an Ansel sheath was placed above the total left common iliac occlusion. The total iliac occlusion was crossed easily with a 0.035 -inch wire. A $4 \mathrm{~F}$ glide catheter was then guided past the occlusion over the 0.035 -inch wire to the left common femoral artery and further angiograms taken through the glide catheter positioned in left common femoral to evaluate for distal arterial anatomy past the left common iliac occlusion. The superficial femoral artery (SFA) on the left side was occluded proximally but the entire popliteal was filling via PFA collaterals (Figure 2). Further distal imaging, below the knee, revealed a three-vessel run off to the foot even though with slow flow. The SFA was also crossed with a 0.035 -inch wire and endovascular intervention initiated with intravenous heparin used as the anticoagulant.

Balloon angioplasty was done over the occluded Iliac and SFA segments using a $4 \mathrm{~mm}$ by $120 \mathrm{~mm}$ balloon catheter. The iliac and SFA still looked occluded despite the angioplasty and below the knee vessels became angiographically worse, looking severely and diffusely diseased on angiography due to further distal embolization.

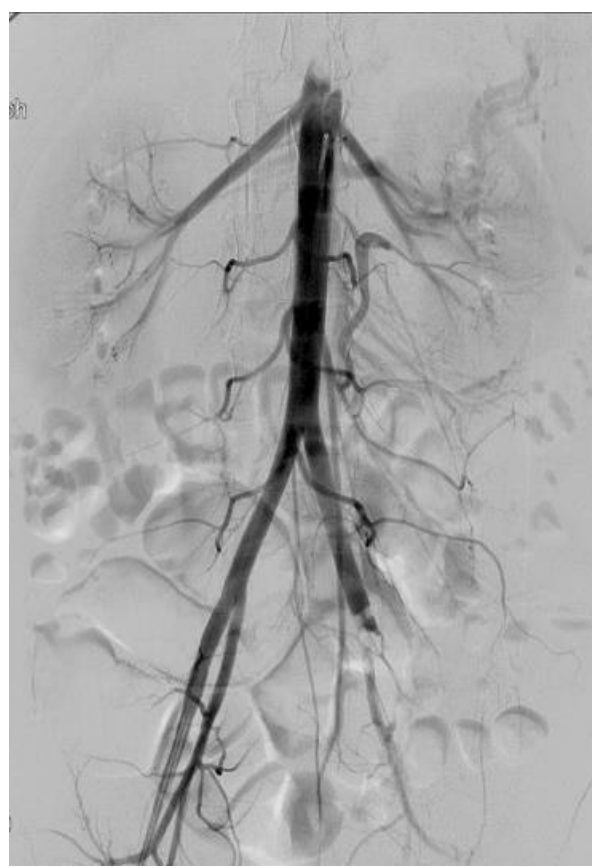

Figure 1. Left leg: Occluded left common iliac artery due to septic embolism with no distal flow

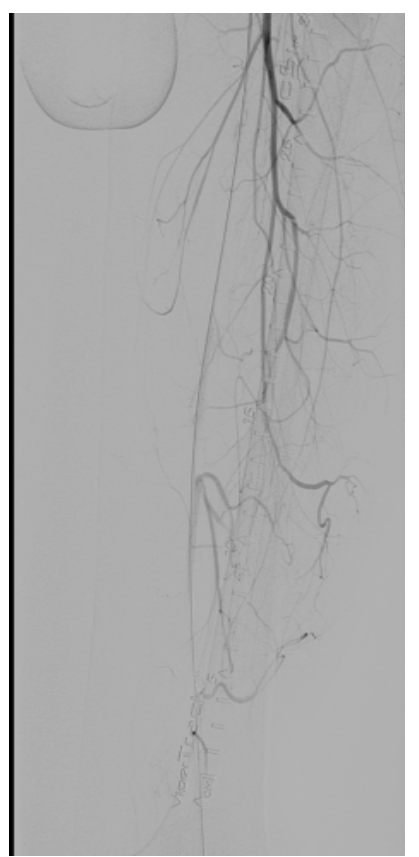

Figure 2. Left Leg: Digital subtraction angiography with catheter tip in common femoral artery showing profunda femoris collaterals and no flow in superficial femoral artery

Ultrasound enhanced catheter directed thrombolysis (Ekos corporation) device was then placed into the left lower extremity over the wire extending from left iliac to distal left SFA and $24 \mathrm{mg}$ of tissue plasminogen activator (tPA) was infused for twelve hours overnight (Figure 3). Repeat angiography through the Ekos sheath showed a marked improvement with patent iliac and SFA even though the SFA had a residual focal lesion proximally (Figure 4). However, there was a new occlusion of the proximal PFA due to embolization related to thrombolysis (Figure 5). The PFA was then crossed with the 0.035 inch wire at the site of the proximal occlusion and Rheolytic thrombectomy done to the PFA followed by balloon angioplasty to both PFA and SFA. The PFA stayed occluded and the SFA still had severe stenosis. The balloon angioplasty helped the proximal SFA but once again was unsuccessful for proximal PFA occlusion. The Rheolytic thrombectomy was tried also on the PFA but the PFA stayed occluded so aspiration thrombectomy was considered for the PFA persistent occlusion.

Hence, multiple aspiration thrombectomy procedures were performed to physically remove the embolized vegetations from the PFA. For this purpose, a 0.014-inch wire was used to wire the PFA. For PFA, $6 \mathrm{~F}$ and later 7F Multipurpose guiding catheters were used for aspiration of the embolic material by placing a large syringe with a stopcock to create negative pressure. The 6 French crossover Ansel sheath had been replaced over a 0.035 wire for a $7 \mathrm{~F}$ Ansel sheath and then 7F Multipurpose guide was used for aspiration keeping the large size of embolized vegetations in mind that needed a large bore aspiration catheter. Aspiration thrombectomy led to retrieval of pieces of embolized material that were sent to pathology laboratory.

Then, angiography of the left leg revealed successful aspiration thrombectomy results with completely patent PFA and SFA vessels. However, at that time, it was realized that there was worsened distal disease below the knee with poor flow and no runoff reaching the left foot, again due to further distal embolization and spasm. Then, aspiration thrombectomy was done in below the knee vessels with the same 7F Mulitpurpose guide with the same technique as described above leading to one vessel run off to the left foot (Figure 6), a result 
Kang K (2020) Ultrasound assisted catheter directed thrombolysis and aspiration thrombectomy for acute limb ischemia caused by embolization of infective endocarditis vegetations

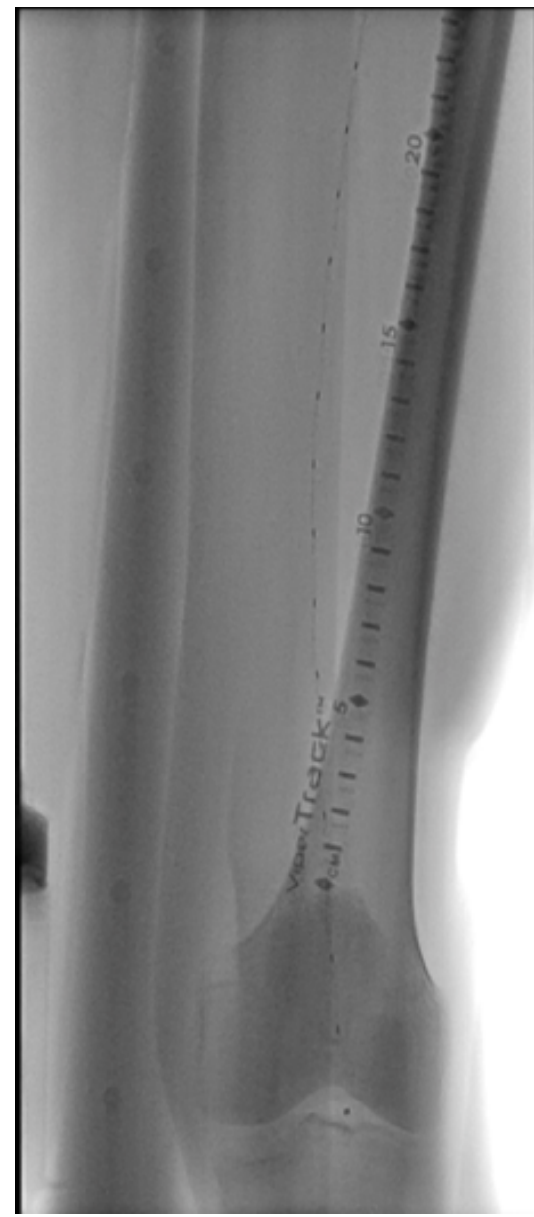

Figure 3. Left Leg: Ekos catheter seen in the iliac artery and the superficial femoral artery with the tip of the catheter reaching the popliteal artery

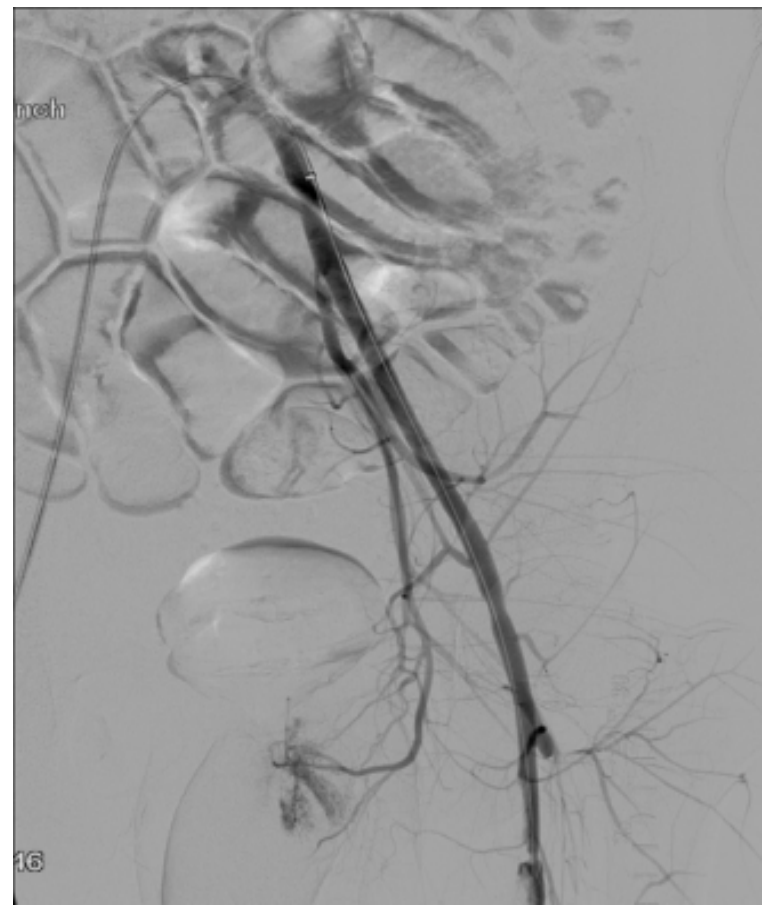

Figure 4. Left Leg: Patent left iliac and patent superficial femoral artery (SFA) with a residual focal lesion in proximal SFA. Profunda femoris has new proximal occlusion

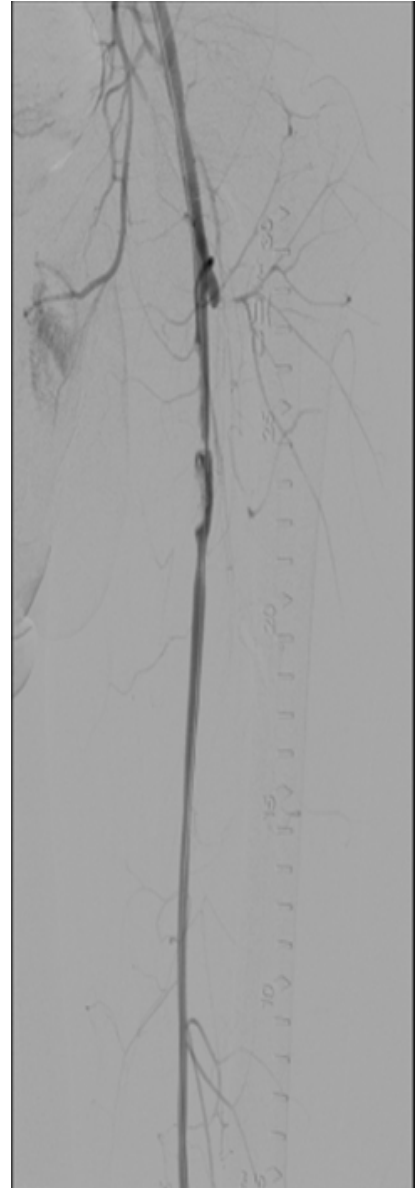

Figure 5. Left leg: Patent superficial femoral artery (SFA) after overnight thrombolysis with a residual focal non-flow limiting lesion in proximal SFA. Profunda femoris has new proximal occlusion

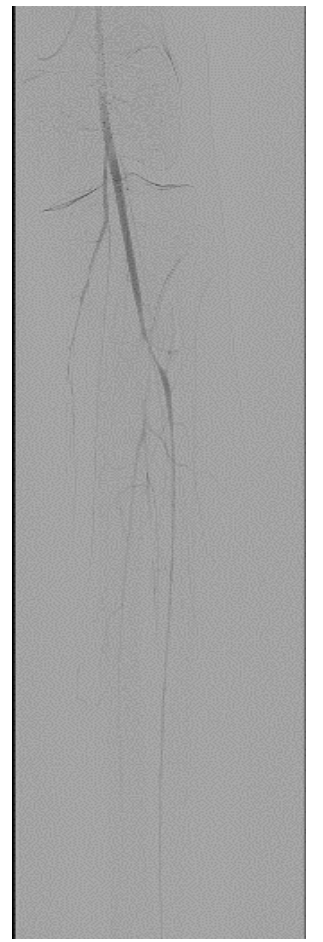

Figure 6. Left leg: Patent one vessel run off to the foot over the left leg-final results 
Kang K (2020) Ultrasound assisted catheter directed thrombolysis and aspiration thrombectomy for acute limb ischemia caused by embolization of infective endocarditis vegetations

that was accepted as final as the patient was asymptomatic by then and with palpable left pedal pulses.

The right leg was targeted next. It had proximal PFA occlusion and distal below the knee occlusion and the access was obtained from the left common femoral artery. Occluded proximal PFA and distal popliteal on the right side were confirmed (Figure 7). There were distal collaterals below the knee leading to filling of the anterior tibial artery. The occluded PFA was crossed with a 0.014-inch wire and treated with $4 \mathrm{~mm}$ balloon angioplasty catheter but once again with no angiographic improvement with balloon angioplasty. The occlusion was then treated with aspiration thrombectomy using a $6 \mathrm{~F}$ Multipurpose guiding catheter with the same technique as on the left leg. There was increased patency of the PFA as a result of this. The distal popliteal occlusion was similarly crossed with a wire and treated with a balloon catheter. Again, no improvement was seen below the knee using balloon angioplasty and noting the results from the left leg where ultrasound facilitated thrombolysis had yielded good results in achieving vessel patency, overnight Ekos catheter directed thrombolytic therapy was applied with the Ekos catheter tip in the proximal peroneal artery (Figure 8). The patient was noted to markedly improve upon angiographic visualization. The PFA was patent with residual stenotic lesions but looking markedly improved compared to the total occlusion from the day before. The SFA and popliteal were patent and below the knee, there was single vessel run off to the posterior tibial artery that had moderate residual lesions only (Figure 9). Then, the lower extremity procedures were concluded, and the patient no longer had any further leg discomfort. The retrieved pieces from the aspiration thrombectomy on pathological reports were consistent with septic emboli (Figure 10).

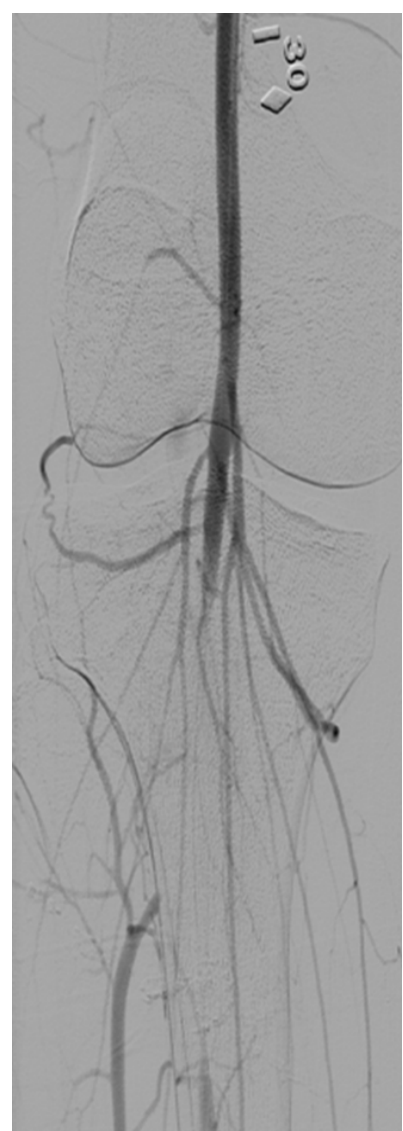

Figure 7. Right leg: Filling defect in right distal popliteal artery with collaterals filling the anterior tibial artery

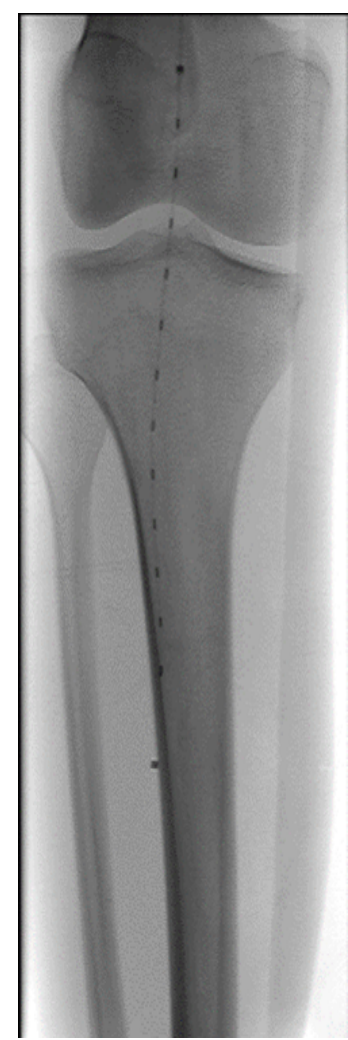

Figure 8. Right leg: Ekos catheter with the tip ending in the peroneal artery

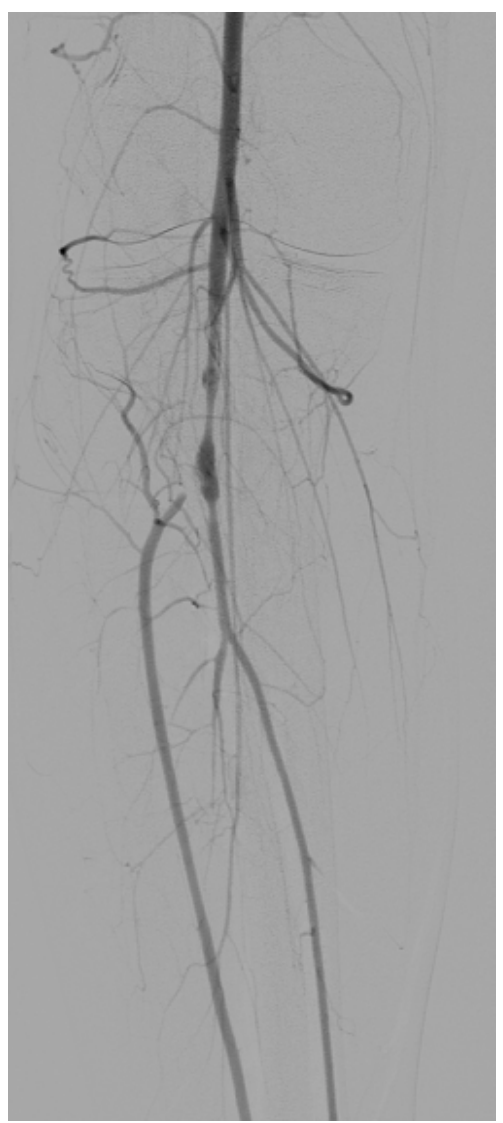

Figure 9. Right leg: Patent popliteal and tibioperoneal trunk after overnight thrombolysis with moderate residual lesions 
Kang K (2020) Ultrasound assisted catheter directed thrombolysis and aspiration thrombectomy for acute limb ischemia caused by embolization of infective endocarditis vegetations

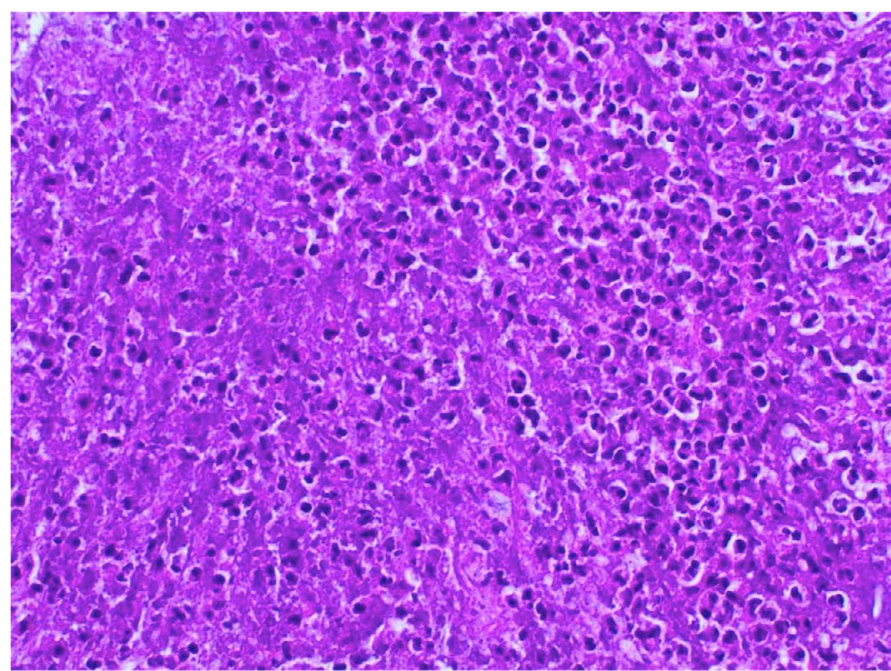

Figure 10. High power view of thrombus material showing sheets of neutrophils and necrotic cellular debris (Hematoxylin and eosin stain, 400X magnification)

He had mitral valve replacement and upon 6 months of follow up in outpatient physician office setting had no further lower extremity ischemic symptoms or signs.

\section{Conclusion}

IE is a relatively common condition [1]. Lower limb embolization is seen in minority (5-8\%) of cases with septic emboli from IE. Antiplatelet or anticoagulant therapy has not been shown to reduce the risk of embolic risk in IE. The risk of IE is lowered after a week of antimicrobial therapy, but no percutaneous interventional therapy has an established role in the treatment of septic emboli. The best treatment, percutaneous or surgical for lower extremity septic emboli unresponsive to medical therapy is not known $[1,4]$. However, surgical treatments have been performed most often for recalcitrant septic emboli despite significant morbidity and mortality [4].

Our patient presented with ALI within a day of his diagnosis and the emboli with IE can occur before diagnosis, during therapy, or after therapy is completed $[1,4]$. Septic emboli from IE are often not considered as responsive to thrombolytics or even anticoagulation because of the infected vegetation status rather than a pure clot status of these emboli and the possibility of catastrophic bleeding from mycotic aneurysms [4]. However, even the septic emboli, vegetations start as platelet and fibrin deposition and hence, thrombolytic treatments may be effective especially if given at reduced doses with catheter directed techniques rather than at higher doses with systemic intravenous therapy. Our patient was considered a poor surgical candidate for lower extremity vascular surgery considering his severe cardiac disease and underlying frail status with very Body Mass Index and underlying Facioscapulohumeral dystrophy as documented by his evaluating cardiologist. An attempt was, henceforth, made to try percutaneous methods including novel approaches like Ekos thrombolysis, which are not standard of care for septic emboli causing ALI but could work given the partial fibrin composition of such lesions.

However, percutaneous treatments are indeed the standard of care for non-infectious emboli that cause ALI $[2,4]$. Overall, the most common causes of ALI in patients are not septic emboli from IE but thrombotic proximal arterial or cardiac emboli that lead to arterial occlusion creating a "leg attack" that is a true emergency. If the limb remains ischemic for more than about 6 hours, permanent motor and sensory losses could result. The standard of care of treating ALI due to such non-septic emboli is thrombectomy techniques using percutaneous intervention. These include thrombectomy with catheter directed thrombolytic therapy or percutaneous mechanical thrombectomy (with techniques like Rheolytic or aspiration thrombectomy). So, standard techniques recommended for thrombotic non-infectious emboli were applied to our patient who was different because his emboli were from large infected vegetations from a myxomatous mitral valve rather than most common proximal thrombotic sources that cause ALI that is atrial fibrillation or aneurysmal atherosclerotic arteries.

Many different modalities of percutaneous treatment had to be tried over 4 days on our patient as he had recalcitrant embolic burden from the IE vegetations in both limbs. Initial attempts were made with balloon angioplasty, which in our patient were uniformly unsuccessful in attaining angiographic improvement. Rheolytic thrombectomy was attempted but not with successful results. Angiographic success was achieved with actual retrieval of septic embolic material to the outside of the body with aspiration thrombectomy when done with large lumen guiding catheters. The lack of response to Rheolytic thrombectomy testifies to the possible lack of response of endocarditis embolized vegetations that were more likely to consist of chronic and organized clots rather than fresh clots that would be broken up by Rheolytic thrombectomy alone. The Rheolytic thrombectomy may work better in clots that have formed within 48 hours [2,4]. Aspiration thrombectomy led to retrieval of pieces of embolized material that were evaluated by pathology laboratory in our hospital and reported as over $1.4 \mathrm{~cm}$ in size and consistent with septic emboli. However, greatest angiographic and clinical success was achieved by ultrasound assisted catheter directed thrombolysis that led to improvement each time it was used for twelve hours of duration overnight. Ultrasound enhanced local thrombolysis has been noted to have measurable benefits in treatment of acute thrombotic or embolic occlusion but has not been tested in septic emboli [5].

Septic emboli can occur in patients with Infective Endocarditis and occasionally, the septic embolic can cause acute limb ischemia (ALI). Percutaneous approach with ultrasound facilitated thrombolysis (Ekos corporation) and aspiration thrombectomy methods can treat ALI originating from septic emboli. The best response in our patient appeared to result from thrombolysis possibly because of underlying fibrin and platelet components of vegetations. Aspiration thrombectomy also appeared to improve angiographic outcomes in our patient but because of the large size of embolized vegetations often encountered, large bore catheters are recommended. An option to reduce distal emboli may have been to use distal protection during these procedures but it was difficult to see any landing zones for these devices. We sent the pieces that we extracted from aspiration thrombectomy to the pathology laboratory and the pathologist reported the pieces as organized thrombus with acute inflammatory exudate, which was consistent with septic emboli. Balloon angioplasty techniques appeared unsuccessful in our patient repetitively and may not be effective in isolation for septic emboli.

\section{Declarations}

- Ethics approval and consent to participate 'Not applicable'

- Consent for publication 'Not applicable'

- Availability of data and material 'Not applicable' This patient's data is available in UPMC Hamot Hospital

- Competing interests The authors have no competing interests. 
Kang K (2020) Ultrasound assisted catheter directed thrombolysis and aspiration thrombectomy for acute limb ischemia caused by embolization of infective endocarditis vegetations

- Funding None

- Authors' contributions All authors contributed to the Case Report significantly and all authors have read and approved the final manuscript.

- Acknowledgements 'Not applicable'

\section{References}

1. Mann DL,Zipes DP,Libby P,Bonow R(2015)Braunwald's HeartDisease. A Textbook of Cardiovascular Medicine. Philadelphia, Pa: W.B. Saunders, pp: 1524-1550.
2. Rajan DK, Patel NH, Valji K, Cardella JF, Brown DB, et al. (2009) Quality improvement guidelines for percutaneous management of acute limb ischemia. $J$ Vasc Interv Radiol 20: 208-218.

3. Pessinaba S, Kane Ad, Ndiaye MB, Mbaye A, Bodian M, et al. (2012) Vascular complications of infective endocarditis. Med Mal Infect 5: 213-217.

4. Mareddy R, George J (2013) Endovascular Management of Acute Limb Ischemia from Septic Emboli. Vascular Disease Management 10:71-74.

5. Wissgott C, Richter A, Kamusella P, Steinkamp HJ (2007) Treatment of critical limb ischemia using ultrasound-enhanced thrombolysis (PARES Trial): final results. $J$ Endovas Ther 14: 438-443.

Copyright: $\odot 2020$ Kang K. This is an open-access article distributed under the terms of the Creative Commons Attribution License, which permits unrestricted use, distribution, and reproduction in any medium, provided the original author and source are credited. 